\title{
Dystrophin deregulation is associated with tumor progression in KIT/PDGFRA mutant gastrointestinal stromal tumors
}

\author{
Maria A Pantaleo ${ }^{1,2^{*}}$, Annalisa Astolfi ${ }^{2}$, Milena Urbini ${ }^{2}$, Fabio Fuligni ${ }^{1}$, Maristella Saponara ${ }^{2}$, Margherita Nannini ${ }^{1}$, \\ Cristian Lolli ${ }^{1}$, Valentina Indio ${ }^{2}$, Donatella Santini ${ }^{3}$, Giorgio Ercolani ${ }^{4}$, Giovanni Brandi ${ }^{1}$, Antonio D Pinna ${ }^{4}$ \\ and Guido Biasco ${ }^{1,2}$
}

\begin{abstract}
Background: Intragenic deletions of the dystrophin-encoding and muscular dystrophy-associated DMD gene have been recently described in gastrointestinal stromal tumor (GIST), rhabdomyosarcoma (RMS) and leiomyosarcoma (LMS). We evaluated the copy numbers and gene expression levels of DMD in our series of GIST patients who were already studied with wide genome assays, to investigate more fully a correlation between dystrophin status and disease annotations.

Findings: Our study highlighted a recurrent intragenic deletion on chromosome $X$, involving the DMD gene that codes for human dystrophin in GIST patients. Of 29 KIT/PDGFRA mutant GIST samples, 9 (31\%) showed deletions of the DMD gene, which were focal and intragenic in 8 cases, and involved loss of an entire chromosome in one case (GIST_188). DMD loss was seen in only 5 patients with metastasis, whereas 18 out of 20 patients with localized disease had wild-type DMD ( $P=0.0004$, Fisher exact test). None of the 6 KIT/PDGFRA WT GIST showed DMD alterations.

Conclusions: Our study confirms the presence of DMD deletions only in KIT/PDGFRA mutant GIST and this event is almost associated with metastatic disease. These findings are, of course, quite preliminary but support development of potential therapeutic strategies that target and restore DMD function in the treatment of metastatic GIST.
\end{abstract}

Keywords: Dystrophin, DMD, Gastrointestinal stromal tumors, KIT/PDGFRA wild type

\section{Findings}

Intragenic deletions of the dystrophin-encoding and muscular dystrophy-associated $D M D$ gene have been recently described in many common human mesenchymal tumors, including gastrointestinal stromal tumor (GIST), rhabdomyosarcoma (RMS) and leiomyosarcoma (LMS) which show myogenic differentiation [1]. In particular, $D M D$ deletions were found in 19 of 29 GIST tumors, 3 of 4 RMS tumors and 3 of 7 LMS tumors. Moreover, DMD deletions and their protein expression were not found in nonmyogenic cancers, or in benign counterparts of GIST, RMS and LMS. In GIST, dystrophin is downregulated in metastatic GIST and primary high-risk GIST, but not in

\footnotetext{
*Correspondence: maria.pantaleo@unibo.it

'Department of Specialized, Experimental and Diagnostic Medicine, Sant'Orsola-Malpighi Hospital, University of Bologna, Bologna, Italy

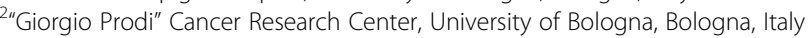
Full list of author information is available at the end of the article
}

low-risk GIST, which implies this downregulation is a late event in progression of this disease. Finally, dystrophin inhibits myogenic sarcoma cell migration, invasion, anchorage independence and invadopodia formation; and when deregulated, dystrophin restoration inhibits invasiveness and migration in sarcoma cell lines. These data validate dystrophin as a tumor suppressor and likely anti-metastatic factor. In light of these findings, we evaluated $D M D$ copy number and gene expression levels in our series of GIST patients who had already been studied with wide genome assays, to investigate more fully the correlation between dystrophin status and disease annotations.

\section{Patient selection and tumor sample collection}

Dystrophin status was evaluated using already-available data from wide genome assays done on tumor specimens collected during surgery and immediately frozen. 
We included samples from 29 patients with mutant KIT/PDGFRA GIST and 6 with wild-type (WT) KIT/ PDGFRA GIST. Among the KIT/PDGFRA WT GIST group, 4 cases were SDH deficient and 2 cases were quadruple KIT/PDGFRA/SDH/BRAF-KRAS-NF1 WT. Out of all 35 patients, 19 had already been reported [2]. Table 1 shows patients' clinical and molecular data. The genomic analysis study was approved by local Ethical Committee.

\section{Copy number analysis}

Genomic DNA was extracted with QiaAmp DNA mini kit (Qiagen, Milan, Italy), labelled and hybridized to SNP array Genome Wide SNP 6.0 (Affymetrix) following

Table 1 Clinical and molecular data of the patients included in the study

\begin{tabular}{|c|c|c|c|c|c|c|c|c|}
\hline ID & Sex & Age & DMD & Start & End & Site & Disease status & KIT/PDGFRA Mutational status \\
\hline GIST_18 & $M$ & NA & loss & ex2 & ex7 & NA & NA & KIT exon 11 V559G \\
\hline \multirow[t]{2}{*}{ GIST_20 } & $M$ & 38 & loss & ex2 & & Small intestine & Metastatic & KIT exon 11 del MYEQW552-557 Z; \\
\hline & & & & ex8 & ex17 & & & KIT exon 18 A829P \\
\hline GIST_22 & $\mathbf{F}$ & 76 & loss & ex1 & ex44 & Stomach & NA & PDGFRA exon 18 D842V \\
\hline GIST_25 & M & 84 & loss & ex1 & ex17 & NA & NA & KIT exon 11 del WKV557-559 F \\
\hline GIST_26 & $M$ & 49 & loss & ex1 & ex11 & Stomach & Metastatic & PDGFRA exon 12 V561D \\
\hline GIST_27 & M & 52 & loss & ex1 & & NA & NA & KIT exon 11 del KV558-559 N \\
\hline GIST_131 & M & 58 & loss & ex3 & ex7 & Small intestine & Metastatic & KIT exon 11 del V569_Y578 \\
\hline GIST_174 & M & 59 & loss & ex1 & ex7 & Stomach & Metastatic & KIT exon 11 L576P \\
\hline GIST_188 & $\mathbf{F}$ & 57 & loss & $\begin{array}{l}\text { All } \\
\text { chrom }\end{array}$ & osome & Small intestine & Metastatic & KIT exon 11 N564_L576del; KIT exon 17 p.N822K \\
\hline GIST_02 & $\mathrm{F}$ & 85 & wt & - & - & Stomach & Localized & KIT exon 11 V560D \\
\hline GIST_04 & M & 79 & wt & - & - & Stomach & Localized & KIT exon 9 ins AY502-503 \\
\hline GIST_05 & M & 68 & wt & - & - & Stomach & Localized & PDGFRA exon 12 ins/del SPDGHE566-571RIQ \\
\hline GIST_08 & M & 62 & wt & - & - & Stomach & Localized & KIT exon 11 V559D \\
\hline GIST_09 & M & 54 & wt & - & - & Stomach & Localized & KIT exon 11 ins TQLPYDHKWEFP574-585 at P585 \\
\hline GIST_11 & M & 65 & wt & - & - & Stomach & Localized & KIT exon 11 del WK557-558 \\
\hline GIST_12 & $\mathrm{F}$ & 66 & wt & - & - & Stomach & Localized & PDGFRA exon 14 K646E \\
\hline GIST_13 & M & 46 & wt & - & - & Small intestine & Localized & KIT exon 11 V559D \\
\hline GIST_14 & M & 56 & wt & - & - & Stomach & Metastatic & KIT exon 11 hom. del WK557-558 \\
\hline GIST_15 & $\mathrm{F}$ & 64 & wt & - & - & Stomach & Localized & PDGFRA exon 18 del DIMH842-845 \\
\hline GIST_16 & $\mathrm{F}$ & 62 & wt & - & - & Stomach & Localized & KIT exon $11 \mathrm{~L} 576 \mathrm{P}$ \\
\hline GIST_121 & M & 72 & wt & - & - & Stomach & Localized & KIT exon 11 V559D \\
\hline GIST_124 & M & 72 & wt & - & - & Stomach & Localized & KIT exon 11 ins $1765-1766$ \\
\hline GIST_125 & $\mathrm{F}$ & 49 & wt & - & - & Stomach & Localized & KIT exon 11 W557R \\
\hline GIST_129 & M & 60 & wt & - & - & Stomach & Localized & KIT exon 11 del/ins Y553-V559L \\
\hline GIST_130 & $\mathrm{F}$ & 79 & wt & - & - & Stomach & Localized & KIT exon 9 ins A502_Y503 \\
\hline GIST_134 & $\mathrm{F}$ & 65 & wt & - & - & Stomach & Localized & KIT exon 11 homoz. V559D \\
\hline GIST_135 & $\mathrm{F}$ & 60 & wt & - & - & Stomach & Localized & KIT exon 11 del W557_E561 \\
\hline GIST_136 & M & 76 & wt & - & - & Stomach & Localized & PDGFRA exon 18 D842V \\
\hline GIST_150 & $\mathrm{F}$ & 56 & wt & - & - & Stomach & Metastatic & KIT exon 11 P551_E554del \\
\hline GIST_07 & $\mathrm{F}$ & 27 & wt & - & - & Stomach & Metastatic & KIT/PDGFRA wild-type (SDH deficient) \\
\hline GIST_10 & M & 29 & wt & - & - & Stomach & Metastatic & KIT/PDGFRA wild-type (SDH deficient) \\
\hline GIST_21 & $\mathrm{F}$ & 25 & wt & - & - & Stomach & NA & KIT/PDGFRA wild-type (SDH deficient) \\
\hline GIST_24 & $\mathrm{F}$ & 18 & wt & - & - & Stomach & Metastatic & KIT/PDGFRA wild-type (SDH deficient) \\
\hline GIST_127 & $\mathrm{F}$ & 63 & wt & - & - & Ileum & Localized & Quadruple wild-type (KIT/PDGFRA/SDH/BRAF-KRAS-NF1 wild-type). \\
\hline GIST_133 & M & 57 & wt & - & - & Duodenum & Localized & Quadruple wild-type (KIT/PDGFRA/SDH/BRAF-KRAS-NF1 wild-type). \\
\hline
\end{tabular}


manufacturer's instructions. Quality control was performed by Contrast QC and MAPD calculation. Copy number analysis was performed by Genotyping Console and visualized with Chromosome Analysis Suite Software (Affymetrix). Hidden Markov Model algorithm was used to detect amplified and deleted segments.

\section{RNA sequencing}

Whole-transcriptome sequencing was performed on RNA isolated from fresh-frozen tumor tissue with the RNeasy spin-column method (Qiagen). Whole-transcriptome RNA libraries were prepared in accordance with Illumina's TruSeq RNA Sample Prep v2 protocol (Illumina, San Diego, California). Paired-end libraries were sequenced at $2 \times 75$ bp read length using Illumina Sequencing by synthesis (SBS) technology. Averages of 85 million reads per sample were analyzed. Mapping on the human reference genome was done with TopHat/BowTie software, while expression level of the $D M D$ gene was expressed as number of mapped reads.

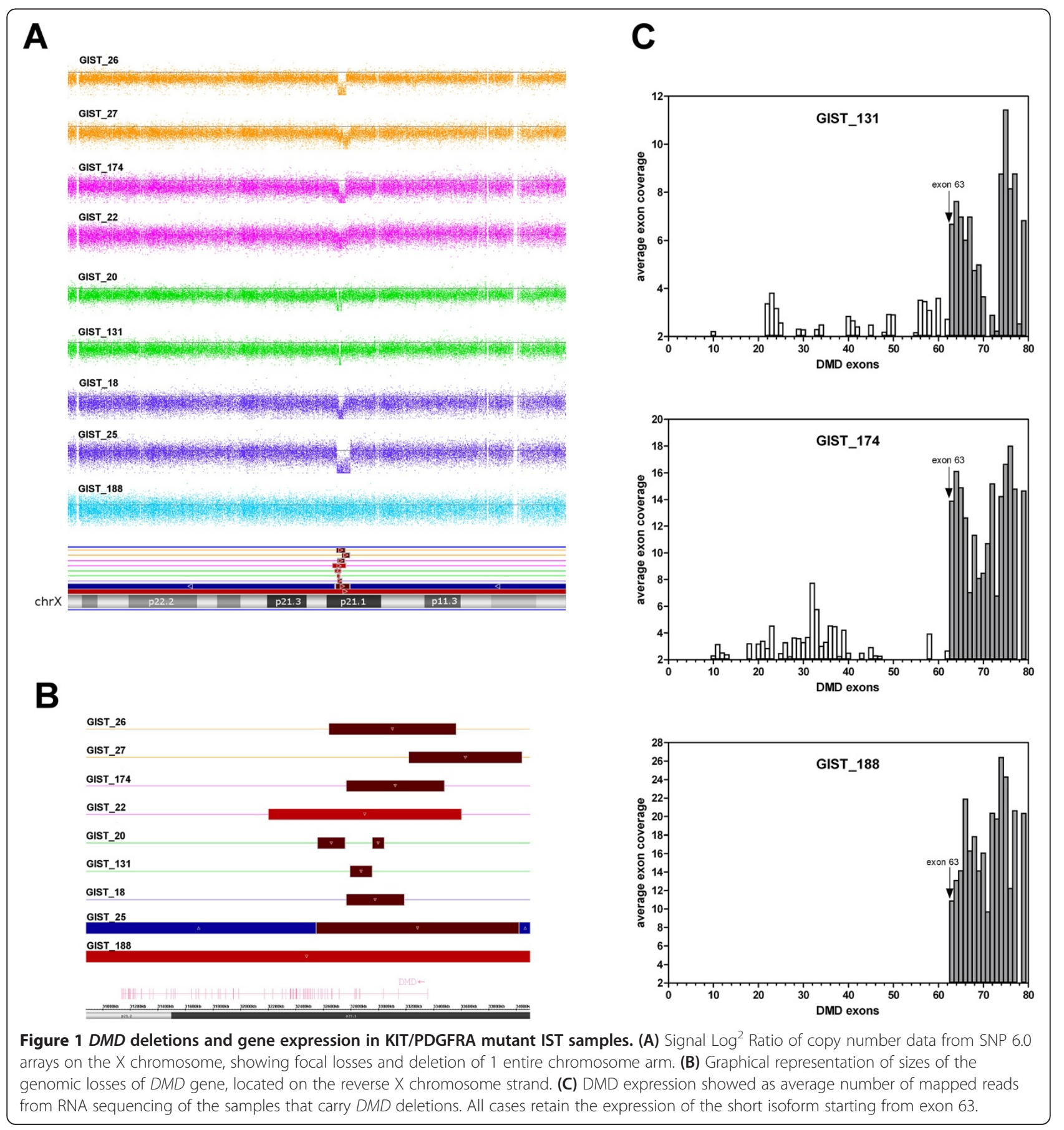




\section{Results and discussion}

The genome-wide analysis of our series highlighted a recurrent intragenic deletion on chromosome $\mathrm{X}$ for the $D M D$ gene, which codes for human dystrophin. Nine of the 29 KIT/PDGFRA mutant GIST (31\%) showed $D M D$ gene deletions (Figure 1A), which were focal and intragenic in 8 cases, and involved loss of a whole chromosome in one case (GIST_188; Figure 1B). None of the 6 KIT/PDGFRA WT GIST samples had $D M D$ alterations.

As $D M D$ is an $\mathrm{X}$-linked gene, deletions were nullizygous in males and heterozygous in females. All focal events involved the $5^{\prime}$ portion of the gene, with the region between exon 2 and exon 7 as the most recurrently involved, and an average deletion size of $770 \mathrm{Mb}$.

RNA-sequencing performed on 3 out of 9 cases with deletions showed that the genomic losses abrogated expression of the largest $D M D$ transcript, while preserving expression of the short isoform, with a transcription start site at exon 63 (Figure $1 C$ ).

Patients with $K I T / P D G F R A$ mutant GIST tended to have higher frequency of $D M D$ deletions in more advanced cases, as DMD loss was seen only in the 5 patients with metastasis, whereas 18 out of the 20 patients with localized disease wild-type $D M D$ (Table $1 ; P=$ 0.0004, Fisher exact test).

Our study confirms the presence of $D M D$ deletions in KIT/PDGFRA mutant GIST. In particular we also observed that this molecular event is associated with more advanced clinical disease such as metastatic tumors. Although these findings are quite preliminary they suggest potential therapeutic strategies that target and restore $D M D$ function in treating metastatic GIST. Larger studies are necessary to correlate $D M D$ status and specific mutations of KIT/PDGFRA receptors to explore novel therapies for GIST that present primary resistance or that initially responds to imatinib but later progresses [3]. As is well known, molecular mechanisms of secondary resistance are various and heterogeneous, the most common being the acquisition of secondary KIT/ PDGFRA mutations or selection of sub-clones with resistant mutations [4]. In our series, of 7 patients who presented primary mutations in KIT exon 11, 2 also had secondary mutations, in KIT exon 17 or KIT exon 18. The 2 patients with mutations in PDGFRA had them in exon $18 \mathrm{D} 842 \mathrm{~V}$ or exon 12 . The small series does not permit any conclusive considerations on the correlation of DMD involvement with KIT/PDGFRA kinase genotype. But as the clinical use of sunitinib and regorafenib after imatinib failure does not completely cover the molecular landscape of the progressing GIST, a novel approach that targets dystrophin deregulation may have relevance in GIST treatment. Moreover, compared with KIT/PDGFRA mutant GIST, in our series all patients with KIT/PDGFRA WT GIST-including 4 who were SDH deficient and 2 who had quadruple KIT/PDGFRA/ SDH/BRAF-KRAS-NF1 WT did not present dystrophin deregulation, even those with metastasis, which confirms once again that KIT/PDGFRA WT GIST should be considered a distinct disease in molecular background and clinical presentation [5].

\section{Conclusion}

In conclusion, deregulation of dystrophin seems to be associated with GIST progression and more data should be accumulated in order to define it as a therapeutic target.

\section{Abbreviations}

DMD: Dystrophin-encoding and muscular dystrophy-associated; GIST: Gastrointestinal stromal tumors; LMS: Leiomyosarcoma; RMS: Rhabdomyosarcoma.

\section{Competing interests}

The authors declare that they have no competing interests.

\section{Authors' contributions}

PAM conceived of the study, participated in its design and coordination and drafted the manuscript. AA and UM carried out the molecular genetic studies, participated in the sequence alignment and drafted the manuscript. FF and IV participated in the design of the study and performed the statistical analysis. SM, NM, LC and EG participated in the analysis and interpretation of data. SD carried out the molecular genetic studies. BG, PAD and BG revised the study critically for important intellectual content. All authors read and approved the final manuscript.

\section{Acknowledgement}

The present study was supported by AIRC, My First Grant 2013.

\section{Author details}

${ }^{1}$ Department of Specialized, Experimental and Diagnostic Medicine, Sant'Orsola-Malpighi Hospital, University of Bologna, Bologna, Italy. ${ }^{2 " G i o r g i o ~}$ Prodi" Cancer Research Center, University of Bologna, Bologna, Italy. ${ }^{3}$ Pathology Unit, Sant'Orsola-Malpighi Hospital, University of Bologna, Bologna, Italy. ${ }^{4}$ Department of General Surgery and Transplantation, Sant'Orsola-Malpighi Hospital, University of Bologna, 40138 Bologna, Italy.

Received: 20 June 2014 Accepted: 28 July 2014

Published: 9 August 2014

\section{References}

1. Wang $Y$, Marino-Enriquez A, Bennett RR, Zhu M, Shen $Y$, Eilers $G$, Lee JC, Henze J, Fletcher BS, Gu Z, Fox EA, Antonescu CR, Fletcher CD, Guo X, Raut CP, Demetri GD, van de Rijn M, Ordog T, Kunkel LM, Fletcher JA: Dystrophin is a tumor suppressor in human cancers with myogenic programs. Nat Genet 2014, 46:601-606.

2. Astolfi A, Nannini M, Pantaleo MA, Di Battista M, Heinrich MC, Santini D, Catena F, Corless CL, Maleddu A, Saponara M, Lolli C, Di Scioscio $\checkmark$, Formica S, Biasco G: A molecular portrait of gastrointestinal stromal tumors: an integrative analysis of gene expression profiling and high-resolution genomic copy number. Lab Invest 2010, 90:1285-1294.

3. Heinrich MC, Corless CL, Blanke CD, Demetri GD, Joensuu $H$, Roberts PJ, Eisenberg BL, von Mehren M, Fletcher CD, Sandau K, McDougall K, Ou WB, Chen CJ, Fletcher JA: Molecular correlates of imatinib resistance in gastrointestinal stromal tumors. J Clin Oncol 2006, 24:4764-4774 
4. Liegl B, Kepten I, Le C, Demetri GD, Heinrich MC, Fletcher CDM, Corless CL, Fletcher JA: Heterogeneity of kinase inhibitor resistance mechanisms in GIST. J Pathol 2008, 216:64-74.

5. Nannini M, Biasco G, Astolfi A, Pantaleo MA: An overview on molecular biology of KIT/PDGFRA wild type ( ${ }^{\mathrm{WTWT}}$ ) gastrointestinal stromal tumours (GIST). J Med Genet 2013, 50:653-661.

doi:10.1186/2045-3329-4-9

Cite this article as: Pantaleo et al:: Dystrophin deregulation is associated with tumor progression in KIT/PDGFRA mutant gastrointestinal stromal tumors. Clinical Sarcoma Research 2014 4:9.

Submit your next manuscript to BioMed Central and take full advantage of:

- Convenient online submission

- Thorough peer review

- No space constraints or color figure charges

- Immediate publication on acceptance

- Inclusion in PubMed, CAS, Scopus and Google Scholar

- Research which is freely available for redistribution

Submit your manuscript at www.biomedcentral.com/submit
() Biomed Central 